\title{
Impact of adjuvant radiotherapy on the survival of women with optimally resected stage III endometrial cancer in the era of modern radiotherapy: a retrospective study
}

Jenny Ling-Yu Chen ${ }^{1,2,3,4}$, Yu-Sen Huang ${ }^{1,4,5,6^{*}}$, Chao-Yuan Huang ${ }^{2}$, Che-Yu Hsu ${ }^{2,4}$, Keng-Hsueh Lan², Wen-Fang Cheng ${ }^{7}$ and Sung-Hsin Kuo ${ }^{2,4}$

\begin{abstract}
Background: The optimal adjuvant treatment for stage III endometrial cancer in the era of modern radiotherapy remains undefined. We investigated the benefit of adjuvant radiotherapy for women who underwent optimal resection for stage III endometrial cancer in the era of modern radiotherapy.

Methods: We retrospectively reviewed patients with endometrial cancer who were treated between 2010 and 2018. Adjuvant treatment included radiotherapy by modern radiotherapy techniques (intensity-modulated or volumetric modulated arc radiotherapy), chemotherapy, or both. Recurrence-free survival (RFS) and overall survival (OS) were calculated using the Kaplan-Meier method and analyzed via multivariate Cox proportional hazards models.

Results: One hundred sixty-one patients were initially included (52, 9, and 100 with stages IIIA, IIIB, and IIIC cancer, respectively); 154 patients (96\%) received adjuvant therapy. Such adjuvant treatment was associated with improved RFS $(p=0.014)$ and OS $(p=0.044)$ over surgery alone. Adjuvant radiotherapy by modern radiotherapy techniques led to low incidence of acute (25\%) and chronic (7\%) grade $\geq 2$ gastrointestinal toxicity. On univariate analysis, non-endometrioid histology and grade 3 status were associated with higher risks of tumor recurrence and death, whereas adjuvant radiotherapy alone or in combination chemotherapy reduced their risks. On multivariate analysis, non-endometrioid histology was associated with increased recurrence (hazard ratio [HR], 2.95; $p=0.009$ ), whereas adjuvant radiotherapy alone or with chemotherapy was associated with lower recurrence (HR, 0.62; $p=0.042)$. Patients $>60$ years of age $(p=0.038)$ as well as those with endometrioid histology $(p=0.045)$, lymphovascular space invasion ( $p=0.031)$, and $\geq 2$ positive lymph nodes $(p=0.044)$ benefited most from adjuvant radiotherapy.
\end{abstract}

Conclusions: Modern adjuvant radiotherapy (intensity-modulated or volumetric modulated arc radiotherapy) alone or with chemotherapy should be considered for women with optimally resected stage III endometrial cancer.

\footnotetext{
* Correspondence: yusen0814@gmail.com

'Department of Radiology, National Taiwan University College of Medicine, No. 7, Chung-Shan S. Rd, Taipei, Taiwan

${ }^{4}$ National Taiwan University Cancer Center, National Taiwan University College of Medicine, Taipei, Taiwan

Full list of author information is available at the end of the article
}

(c) The Author(s). 2020 Open Access This article is licensed under a Creative Commons Attribution 4.0 International License, which permits use, sharing, adaptation, distribution and reproduction in any medium or format, as long as you give appropriate credit to the original author(s) and the source, provide a link to the Creative Commons licence, and indicate if changes were made. The images or other third party material in this article are included in the article's Creative Commons licence, unless indicated otherwise in a credit line to the material. If material is not included in the article's Creative Commons licence and your intended use is not permitted by statutory regulation or exceeds the permitted use, you will need to obtain permission directly from the copyright holder. To view a copy of this licence, visit http://creativecommons.org/licenses/by/4.0/ The Creative Commons Public Domain Dedication waiver (http://creativecommons.org/publicdomain/zero/1.0/) applies to the data made available in this article, unless otherwise stated in a credit line to the data. 
(Continued from previous page)

Trial registration: ClinicalTrials.gov, NCT04251676. Registered 24 January 2020. Retrospectively registered.

Keywords: Endometrial cancer, Stage III, Adjuvant therapy, Intensity-modulated radiotherapy, Volumetric modulated arc radiotherapy

\section{Background}

Endometrial cancer is the most common malignancy of the female genital tract; moreover, its incidence rate continues to increase [1]. Approximately one-fifth of the patients are diagnosed at an already advanced stage; the 5-year overall survival (OS) rates of those with stage III disease who are able to undergo optimal resection are $70-80 \%$ when adjuvant therapy is administered and $30-40 \%$ when it is not $[2,3]$. The most suitable adjuvant treatment for stage III endometrial cancer remains undefined, including the appropriate adjuvant chemotherapy, radiotherapy, or combined chemoradiotherapy options [4-7]; notably, only a few prospective studies on this topic have been performed $[8,9]$.

Adjuvant external beam radiotherapy (EBRT) is conventionally delivered using multiple conformal fields via three-dimensional conformal radiation therapy; modern radiotherapy techniques such as intensity-modulated radiation therapy (IMRT) and volumetric modulated arc therapy (VMAT) have reduced treatment-related toxicity and are becoming more widely available [10, 11]. IMRT, which has the advantage of integrating a multileaf collimator, rotational fan, cone beam delivery system, and robotic arm linear accelerator, delivers a high radiation dose to the target while minimizing the exposure of the organs at risk. Moreover, the arc-based approach for VMAT delivery is designed to further improve dose distribution through dynamic modulation of the gantry rotation speed, dose rate, and multileaf collimator shaping, thereby generating patterns of intensity modulation that deliver optimal treatment doses to the patient $[12,13]$. Modern radiotherapy techniques are associated with better survival rates than conventional radiation delivery for numerous cancer treatments [14, 15]. This is mostly due to their qualities of riskadaptive dose prescription, increased locoregional tumor control, and decreased radiation-related side effects $[16,17]$. Yet, the merits of modern radiotherapy techniques for adjuvant endometrial cancer are still being investigated.

\section{Materials and methods}

The aim of this study was to investigate the benefit of adjuvant radiotherapy for women who underwent optimal resection for stage III endometrial cancer in the era of modern radiotherapy.

\section{Study design and patient selection}

Patients with surgically staged endometrial cancer treated between 2010 and 2018 within the multiinstitution National Taiwan University Hospital Healthcare System (including the National Taiwan University Hospital, the affiliated Yun-Lin branch, and Hsin-Chu branch) were retrospectively investigated. Staging was performed according to the 2009 International Federation of Gynecology and Obstetrics (FIGO) staging system for endometrial cancer [18]. A total of 1273 patients with surgically staged endometrial cancer were identified, among whom 204 had FIGO stage III. Women who were administered chemotherapy or radiotherapy before surgery, received surgery with a palliative intent, or had sarcoma or carcinosarcoma were excluded; hence, 161 patients with stage III endometrial cancer who underwent optimal resection were ultimately included.

\section{Surgery}

Staging surgery included total abdominal hysterectomy, bilateral salpingo-oophorectomy, peritoneal washings, and either selective or systematic pelvic lymphadenectomy. The para-aortic lymph nodes were sampled or dissected from the area between the aortic bifurcation and inferior mesenteric artery in patients with elevated serum cancer antigen 125 , myometrial invasion $>50 \%$, extrauterine spread, or para-aortic lymph nodes $>1 \mathrm{~cm}$ in diameter as identified on preoperative magnetic resonance imaging (per the Korean Gynecologic Oncology Group 2014 criteria). Bulky nodes were removed by dissection whenever possible. An omental biopsy or omentectomy was performed at the discretion of the individual surgeon based on the extent of disease. The patients had no residual disease by the end of primary surgery; the median number of dissected lymph nodes was 12 (range: $0-40$ ).

\section{Chemotherapy}

Chemotherapy regimens were administered according to the physicians' preferences; the most common treatments were platinum-based regimens with the majority $(81 \%)$ receiving paclitaxel and platinum (carboplatin or cisplatin). Other regimens included cisplatin plus doxorubicin (10\%), and cisplatin plus epirubicin (9\%). The majority of patients received 6 cycles of chemotherapy. Women who received both adjuvant chemotherapy and radiotherapy completed all chemotherapy courses before 
radiotherapy (34\%) or in conjunction with radiotherapy in a sandwich pattern (66\%).

\section{Radiotherapy}

For optimally resected stage III endometrial cancer, the adjuvant radiotherapy was directed at sites of known or suspected tumor involvement, and included EBRT and/ or brachytherapy [19]. All women who underwent adjuvant radiotherapy were treated with EBRT, and vaginal brachytherapy was also used for most (84\%) women. Our health-care system adopted modern radiotherapy including IMRT since 2010 and VMAT since 2015, which helped minimize the dose to the normal organs on the basis of adjuvant radiotherapy [20, 21]. The dose of EBRT was 5040 cGy over 6 weeks, 5 days per week, with daily fractions of $1.8 \mathrm{~Gy}$. Pelvic radiotherapy targeted the common, external, and internal iliac lymph node regions, upper $3 \mathrm{~cm}$ of the vagina, and the paravaginal soft tissue lateral to the vagina in accordance with the updated delineation consensus for gynecologic malignancy [11]. The presacral lymph nodes were irradiated in patients with cervical involvement. In patients with multiple positive pelvic nodes or documented paraaortic lymph node disease, extended-field radiotherapy that encompassed the pelvic volume and also targeted the entire common iliac chain and para-aortic lymph node region was considered. A boost dose of 5-10 Gy was also considered for documented extranodal extension or enlarged unresected lymphadenopathy. An example of a modern radiotherapy technique with its associated isodose curves is shown in Fig. 1. EBRT was performed using $10 \mathrm{MV}$ radiation beams from the Elekta Synergy accelerator (Elekta, Stockholm, Sweden) or the Varian TrueBeam ${ }^{\mathrm{Tm}}$ Radiotherapy System (Varian, Palo a

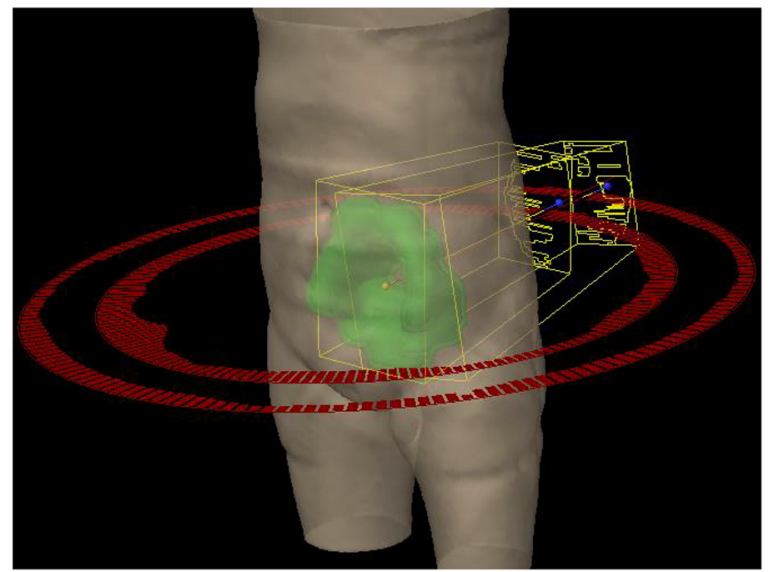

C

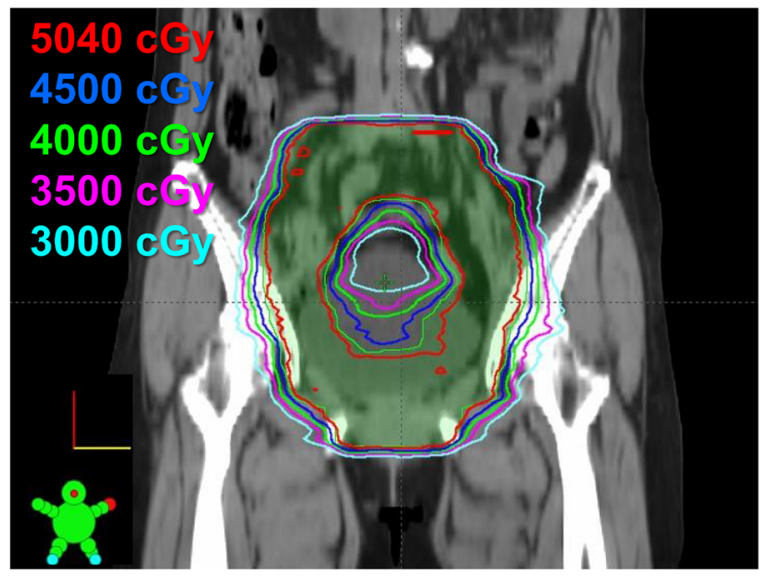

b

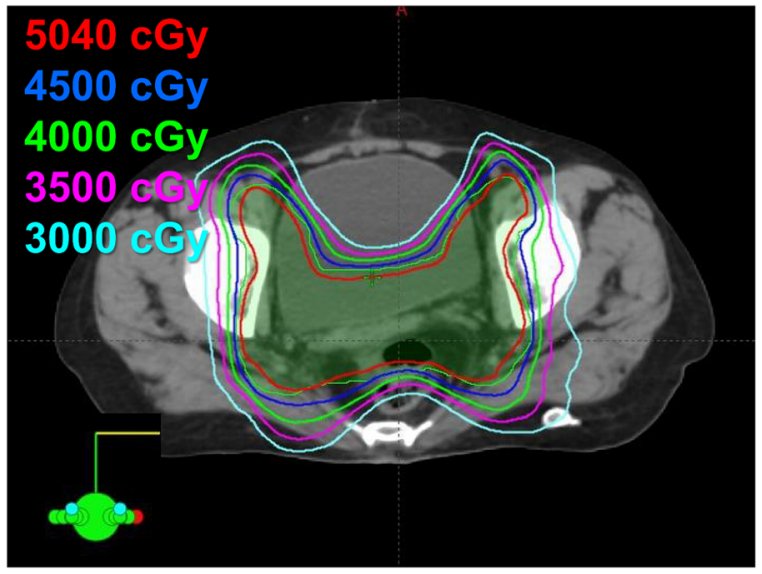

d

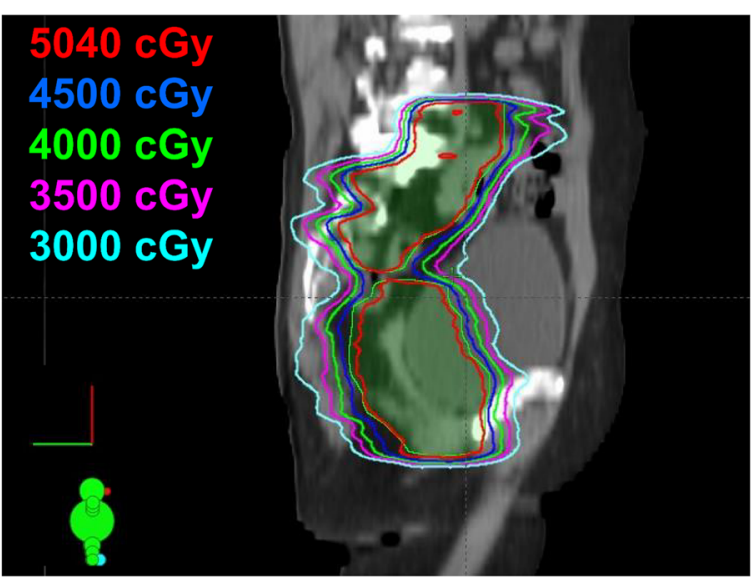

Fig. 1 Modern radiotherapy technique and dose distributions. This figure shows the isodose distributions in a patient with optimally resected stage III endometrial cancer who underwent adjuvant radiotherapy via volumetric modulated arc therapy (VMAT). A 50.4-Gy dose (28 fractions) was prescribed to the target volumes. a Beam arrangement according to the VMAT plan. Dose distributions in the axial (b), coronal (c), and sagittal (d) views. The green color-washed areas indicate the target volume (i.e., vagina and nodal lymphatics in the pelvis). The red, blue, green, pink, and indigo lines represent isodose curves of 50.4, 45, 40, 35, and 30 Gy, respectively. 
Alto, CA, USA) in multiple coplanar ports. The treatment position was verified weekly using cone-beam CT $\mathrm{X}$-ray volume imaging [14]. Following pelvic radiation, high dose-rate brachytherapy via a vaginal cylinder was used to boost the upper two-thirds of the vagina. Brachytherapy doses of 6 Gy per fraction for 2 to 3 fractions prescribed to the vaginal mucosa were used, and they were delivered using the "Nucletron HDR" Ir-192 remote afterloading technique [22, 23].

\section{Statistical analysis}

Statistical analyses were performed using the Statistical Package for Social Sciences for Windows, version 22.0 (IBM, Armonk, NY, USA). Differences in the distributions of variables among the treatment groups were analyzed using the chi-squared test. Acute and late toxicities were rated according to the Common Terminology Criteria for Adverse Events version 4.0. Survival data were confirmed with the Cancer Registry Medical Information Management Office in the health-care system. All patients were followed for at least 3 months for the first 2 years and every 4-6 months thereafter until recurrence or death $[2,23]$. Locoregional recurrence was defined as failure in the pelvic regions or evidence of para-aortic lymphadenopathy below the T12-L1 interspace. Distant metastasis was defined as disease relapse outside the locoregional area as detected pathologically, cytologically, or radiologically. All events were calculated from the date of treatment completion. Analysis was conducted using the follow-up data accumulated as of September 30, 2019. Actuarial estimates of recurrencefree survival (RFS) and OS were calculated using the Kaplan-Meier method and compared using the log-rank test. All prognostic variables found to be significant on univariate analysis were subjected to multivariate analysis using the Cox proportional hazards regression model. A $p$-value $\leq 0.05$ was considered statistically significant.

\section{Results}

\section{Patient characteristics}

A total 161 optimally resected patients were investigated (52 with stage IIIA, 9 with stage IIIB, and 100 with stage IIIC disease); none had residual disease upon completing primary surgery. The patient and tumor characteristics are shown in Table 1 . The median age at diagnosis was 57 years, and the predominant histologic subtype was endometrioid (85\%). Lymphovascular space invasion was present in nearly three-fourths $(74 \%)$ of the tumors, and nearly one-third (32\%) had FIGO grade 3 histology. Positive peritoneal washing cytology was observed in nearly one-fourth $(24 \%)$ of the patients.
Table 1 Patients' demographics and tumor characteristics $(n=161)$

\begin{tabular}{|c|c|}
\hline Age (years) [range] & 57 [33-89] \\
\hline \multicolumn{2}{|l|}{ FIGO stage } \\
\hline$\| I A$ & $52(32)$ \\
\hline$\| I B$ & $9(6)$ \\
\hline IIIC & $100(62)$ \\
\hline \multicolumn{2}{|l|}{ Histology } \\
\hline Endometrioid & $137(85)$ \\
\hline Papillary serous & $16(10)$ \\
\hline Clear cell & $5(3)$ \\
\hline Mucinous & $2(1)$ \\
\hline Neuroendocrine & $1(1)$ \\
\hline \multicolumn{2}{|l|}{ Tumor grade } \\
\hline 1 & $52(32)$ \\
\hline 2 & $57(36)$ \\
\hline 3 & $52(32)$ \\
\hline \multicolumn{2}{|l|}{ Lymphovascular space invasion } \\
\hline Nil & $42(26)$ \\
\hline Present & $119(74)$ \\
\hline \multicolumn{2}{|l|}{ Peritoneal cytology } \\
\hline Negative & $122(76)$ \\
\hline Positive & $39(24)$ \\
\hline \multicolumn{2}{|l|}{ Pelvic LN metastasis } \\
\hline Nil & $66(41)$ \\
\hline Present & $95(59)$ \\
\hline Median number of positive LN [range] & $2[1-11]$ \\
\hline \multicolumn{2}{|l|}{ Para-aortic LN metastasis } \\
\hline Nil & $135(84)$ \\
\hline Present & $26(16)$ \\
\hline Median number of positive LN [range] & $2[1-5]$ \\
\hline \multicolumn{2}{|l|}{ Total LN metastasis } \\
\hline Nil & $61(38)$ \\
\hline Present & $100(62)$ \\
\hline Median number of positive LN [range] & $2[1-12]$ \\
\hline
\end{tabular}

\section{Adjuvant treatments and outcomes}

One hundred fifty-four patients (96\%) received an adjuvant treatment; 67 (42\%) received chemotherapy alone, $29(18 \%)$ received adjuvant radiotherapy alone, and 58 (36\%) underwent chemoradiotherapy. In the 87 patients who received adjuvant radiotherapy with or without chemotherapy, IMRT was used in 45 (52\%) patients and VMAT was used in $42(48 \%)$ patients. Of the remaining 7 patients, 4 decided against adjuvant therapy and 3 had comorbidities that precluded such treatments. The relationships between the patients' characteristics and type 
of postoperative adjuvant treatment received are described in Table 2. There was no significant difference in the type of administered treatment among patients grouped according to FIGO stage, tumor histology, tumor grade, lymphovascular space invasion, positive peritoneal cytology, or number of positive lymph nodes. However, elderly patients were less frequently treated with combined chemoradiotherapy than were their younger counterparts $(p=0.046)$.

Regarding adjuvant radiotherapy-related gastrointestinal side effects, $23 \%$ of patients had acute grade 2 diarrhea, and $2 \%$ had acute grade 3 diarrhea; $5 \%$ had grade 2 late toxicity with moderate diarrhea requiring medications, and $2 \%$ developed grade 3 radiation proctitis that were successfully treated by colonoscopy argon plasma coagulation. None had acute or late grade 4+ gastrointestinal symptoms. After a median follow-up of 44 months (range: $3-103$ months), 140 patients (87\%) were alive, whereas 21 patients (13\%) had died; the majority of deaths (95\%) were attributed to cancer progression. Fifty-one patients had tumor recurrence, which was locoregional in 26 (vaginal stump, pelvic, or para-aortic recurrence) and distant in 41 (lung, liver, bone, brain, distant lymphadenopathy, or peritoneal carcinomatosis); moreover, 16 patients had both locoregional recurrence and distant metastasis. Administration of any type of adjuvant treatment was associated with a longer 5-year RFS (67\% vs. 0\%, $p=0.014$, Supplementary Figure 1a) and OS ( $84 \%$ vs. $66 \%, p=0.044$, Supplementary Figure $1 b)$. The type of adjuvant treatment did not influence the risks of tumor recurrence in patients with optimally resected stage III disease; the 5-year RFS was $61 \%$ in those who underwent adjuvant chemotherapy alone, $73 \%$ in those who received adjuvant radiotherapy alone, and $79 \%$ in those who received combined chemoradiotherapy ( $p=0.172$, Fig. 2a). Likewise, the 5 -year OS was $80 \%$ for patients treated with adjuvant chemotherapy alone, $85 \%$ for those treated with adjuvant radiotherapy alone, and $86 \%$ for those who received combined chemoradiotherapy ( $p=0.390$, Fig. $2 b$ ).

On the univariate analysis, tumor stage (IIIA or IIIB vs. IIIC), presence of lymphovascular space invasion, positive peritoneal washing cytology, or $\geq 2$ positive lymph nodes were not significantly associated with an increased risk of tumor recurrence or death, whereas older age was associated with an increased risk of tumor recurrence (hazard ratio [HR] 2.14, $p=0.008$ ) but not of death (Table 3). Non-endometrioid histology and grade

Table 2 Relationship between treatment type and patient characteristics ( $n=161)$

\begin{tabular}{|c|c|c|c|c|c|}
\hline & $\begin{array}{l}\text { Chemotherapy } \\
(n=67)\end{array}$ & $\begin{array}{l}\text { Radiotherapy } \\
(n=29)\end{array}$ & $\begin{array}{l}\text { Combined chemoradiotherapy } \\
(n=58)\end{array}$ & $\begin{array}{l}\text { No treatment } \\
(n=7)\end{array}$ & $\overline{p \text {-value }}$ \\
\hline Age & & & & & 0.046 \\
\hline$\leq 60$ years & $42(41 \%)$ & $14(14 \%)$ & $43(42 \%)$ & $4(3 \%)$ & \\
\hline$>60$ years & $25(43 \%)$ & $15(26 \%)$ & $15(26 \%)$ & $3(5 \%)$ & \\
\hline FIGO stage & & & & & 0.389 \\
\hline$\| I A$ or IIIB & $30(49 \%)$ & $8(13 \%)$ & $20(33 \%)$ & $3(5 \%)$ & \\
\hline$\| I C$ & $37(37 \%)$ & $21(21 \%)$ & $38(38 \%)$ & $4(4 \%)$ & \\
\hline Histology & & & & & 0.059 \\
\hline Endometrioid & $56(41 \%)$ & $28(20 \%)$ & $49(36 \%)$ & $4(3 \%)$ & \\
\hline Non-endometrioid & $11(46 \%)$ & $1(4 \%)$ & $9(38 \%)$ & $3(12 \%)$ & \\
\hline Tumor grade & & & & & 0.185 \\
\hline $1-2$ & $46(42 \%)$ & $24(22 \%)$ & $35(32 \%)$ & $4(4 \%)$ & \\
\hline 3 & $21(40 \%)$ & $5(10 \%)$ & $23(44 \%)$ & $3(6 \%)$ & \\
\hline Lymphovascular space invasion & & & & & 0.225 \\
\hline Nil & $22(52 \%)$ & $4(10 \%)$ & $15(36 \%)$ & $1(2 \%)$ & \\
\hline Present & $45(38 \%)$ & $25(21 \%)$ & $43(36 \%)$ & $6(5 \%)$ & \\
\hline Peritoneal cytology & & & & & 0.111 \\
\hline Negative & $45(367 \%)$ & $23(19 \%)$ & $47(39 \%)$ & $7(6 \%)$ & \\
\hline Positive & $22(576 \%)$ & $6(15 \%)$ & $11(28 \%)$ & $0(0 \%)$ & \\
\hline$\geq 2$ positive $L N$ & & & & & 0.669 \\
\hline Nil & $38(44 \%)$ & $14(16 \%)$ & $30(34 \%)$ & $5(6 \%)$ & \\
\hline Present & 29 (39\%) & $15(20 \%)$ & $28(38 \%)$ & $2(3 \%)$ & \\
\hline
\end{tabular}

FIGO The International Federation of Gynecology and Obstetrics, LN lymph node ${ }^{a}$ Significance was determined using the chi-squared test 
a

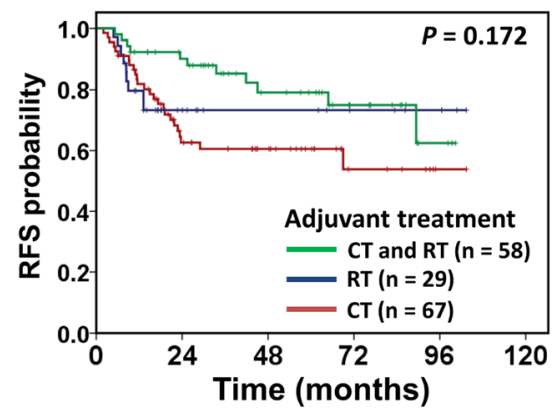

c

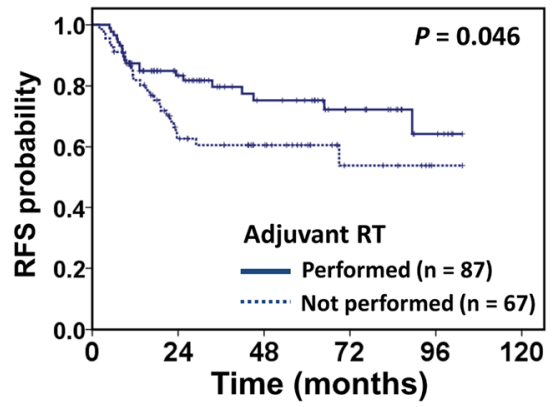

e

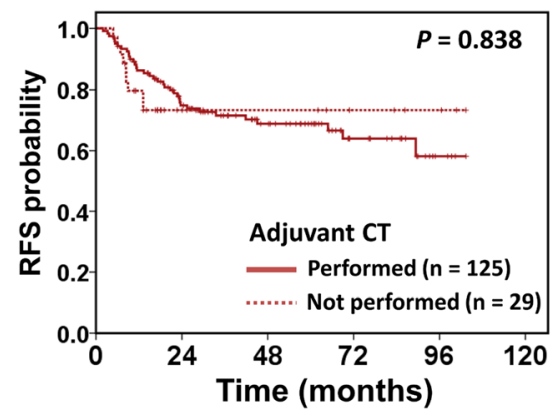

b

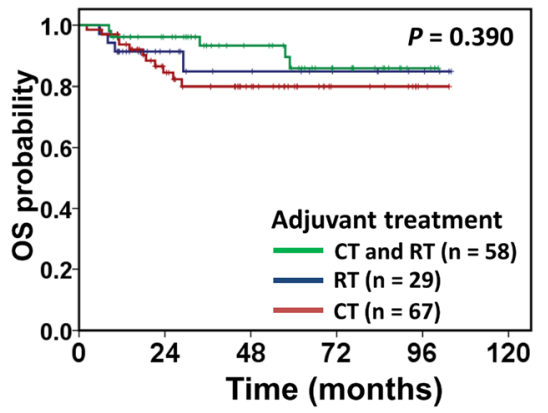

d

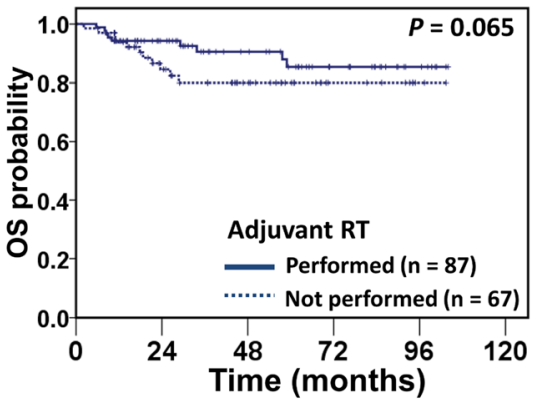

f

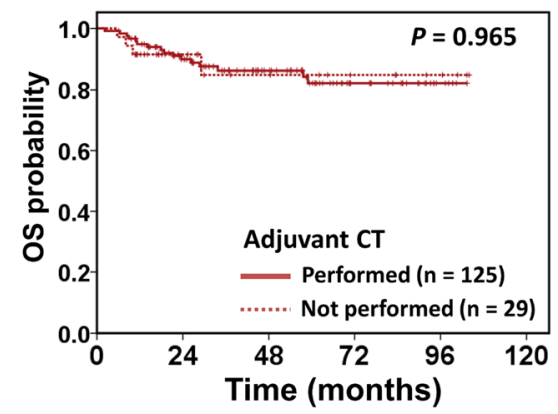

Fig. 2 Survival in patients with optimally resected stage III endometrial cancer by type of adjuvant treatment $(n=154)$. Recurrence-free survival (RFS) (a) and overall survival (OS) (b) of patients based on the type of adjuvant treatment: adjuvant chemotherapy (CT) alone, adjuvant radiotherapy (RT) alone, or combined chemoradiotherapy. RFS (c) and OS (d) of patients based on whether or not they received radiotherapy alone or combined with chemotherapy. RFS (e) and OS (f) of patients based on whether or not they received chemotherapy alone or combined with radiotherapy. $p$-values were determined using Kaplan-Meier log-rank tests.

3 tumor status were associated with increased risks of tumor recurrence (HR: non-endometrioid histology: 5.42; grade 3 tumor status: 1.74 , both $p<0.001$ ) and death (HR: non-endometrioid histology: 4.34, $p=0.001$; grade 3 tumor status: $1.49, p=0.043$ ).

Moreover, the type of adjuvant treatment was associated with clinical outcomes. Patients who received adjuvant radiotherapy alone or combined with chemotherapy experienced a longer 5-year RFS than those who did not receive radiotherapy ( $75 \%$ vs. $61 \%, p=0.046$, Fig. $2 \mathrm{c}$ ) and also showed a trend toward a longer 5 -year OS rate $(85 \%$ vs. $80 \%, p=0.065$, Fig. 2 d). Meanwhile, patients who received adjuvant chemotherapy alone or combined with radiotherapy had similar survival periods as those who did not receive chemotherapy (5-year RFS: 69\% vs. 73\%, $p=$ 0.838, Fig. 2e; 5 -year OS: $82 \%$ vs. $85 \%, p=0.965$, Fig. $2 \mathrm{f}$ ).

On the multivariate analysis, non-endometrioid histology increased the risk of tumor recurrence (HR 2.95, 95\% confidence interval [CI] 1.32-6.61, $p=0.009$ ), whereas adjuvant radiotherapy alone or combined with chemotherapy decreased the risk of tumor recurrence (HR 0.62, 95\% CI 0.31-0.98, $p=0.042$ ) (Table 4).

\section{Effectiveness of adjuvant radiotherapy}

A subgroup analysis was performed to identify patients who might most benefit from adjuvant radiotherapy (Fig. 3). Adjuvant radiotherapy alone or combined with chemotherapy decreased the risk of tumor recurrence 
Table 3 Univariate analysis of potential prognostic factors $(n=154)$

\begin{tabular}{|c|c|c|c|c|c|c|}
\hline & 5-year RFS & HR $(95 \% \mathrm{Cl})$ & $p$-value ${ }^{\mathrm{a}}$ & 5-year OS & HR (95\% Cl) & $p$-value ${ }^{\mathrm{a}}$ \\
\hline Age & & & 0.008 & & & 0.459 \\
\hline$\leq 60$ years & 76 & - & & 86 & - & \\
\hline$>60$ years & 52 & $2.14(1.20-3.79)$ & & 81 & $1.41(0.57-3.51)$ & \\
\hline FIGO stage & & & 0.451 & & & 0.615 \\
\hline$\| I A$ or $\| \mid B$ & 69 & - & & 85 & - & \\
\hline$\| I C$ & 66 & $0.89(0.65-1.21)$ & & 84 & $0.88(0.55-1.43)$ & \\
\hline Histology & & & $<0.001$ & & & 0.001 \\
\hline Endometrioid & 75 & - & & 87 & - & \\
\hline Non-endometrioid & 15 & $5.42(2.88-10.18)$ & & 62 & $4.34(1.63-11.60)$ & \\
\hline Tumor grade & & & $<0.001$ & & & 0.043 \\
\hline $1-2$ & 78 & - & & 88 & - & \\
\hline 3 & 44 & $1.74(1.30-2.32)$ & & 76 & $1.49(1.05-2.35)$ & \\
\hline Lymphovascular space invasion & & & 0.950 & & & 0.232 \\
\hline Nil & 69 & - & & 87 & - & \\
\hline Present & 64 & $1.01(0.71-1.37)$ & & 76 & $1.32(0.83-2.11)$ & \\
\hline Peritoneal cytology & & & 0.174 & & & 0.316 \\
\hline Negative & 70 & - & & 86 & - & \\
\hline Positive & 58 & $1.54(0.82-2.89)$ & & 75 & $1.64(0.62-4.33)$ & \\
\hline$\geq 2$ positive $L N$ & & & 0.545 & & & 0.316 \\
\hline Nil & 69 & - & & 87 & - & \\
\hline Present & 66 & $1.19(0.67-2.12)$ & & 82 & $1.59(0.64-3.95)$ & \\
\hline Adjuvant radiotherapy, alone or combined with chemotherapy & & & 0.046 & & & 0.065 \\
\hline Yes & 75 & $0.62(0.35-0.99)$ & & 85 & $0.53(0.21-1.23)$ & \\
\hline No & 61 & - & & 80 & - & \\
\hline Adjuvant chemotherapy, alone or combined with radiotherapy & & & 0.838 & & & 0.965 \\
\hline Yes & 69 & $1.08(0.52-2.25)$ & & 82 & $1.03(0.34-3.07)$ & \\
\hline No & 73 & - & & 85 & - & \\
\hline Combined chemoradiotherapy & & & 0.084 & & & 0.522 \\
\hline Yes & 81 & $0.48(0.20-1.13)$ & & 87 & $0.67(0.19-2.31)$ & \\
\hline No & 64 & - & & 84 & - & \\
\hline
\end{tabular}

FIGO The International Federation of Gynecology and Obstetrics, LN lymph node, RFS recurrence-free survival, OS overall survival, $H R$ hazard ratio, CI confidence interval

${ }^{a}$ Significance tested using Kaplan-Meier life table analysis and the log-rank test

Table 4 Multivariate analysis of potential prognostic factors $(n=154)$

\begin{tabular}{|c|c|c|c|c|}
\hline & \multicolumn{2}{|c|}{ Recurrence-free survival } & \multicolumn{2}{|l|}{ Overall survival } \\
\hline & $\mathrm{HR}(95 \% \mathrm{Cl})$ & $p$-value ${ }^{a}$ & $\mathrm{HR}(95 \% \mathrm{Cl})$ & $p$-value \\
\hline Age $>60$ years & $1.44(0.75-2.75)$ & 0.277 & $0.93(0.34-2.56)$ & 0.884 \\
\hline Non-endometrioid histology & $2.95(1.32-6.61)$ & 0.009 & $3.27(0.94-11.34)$ & 0.062 \\
\hline Tumor grade 3 & $1.90(0.97-3.75)$ & 0.062 & $1.38(0.48-3.98)$ & 0.555 \\
\hline Adjuvant radiotherapy, alone or combined with chemotherapy & $0.62(0.31-0.98)$ & 0.042 & $0.53(0.20-1.37)$ & 0.188 \\
\hline
\end{tabular}




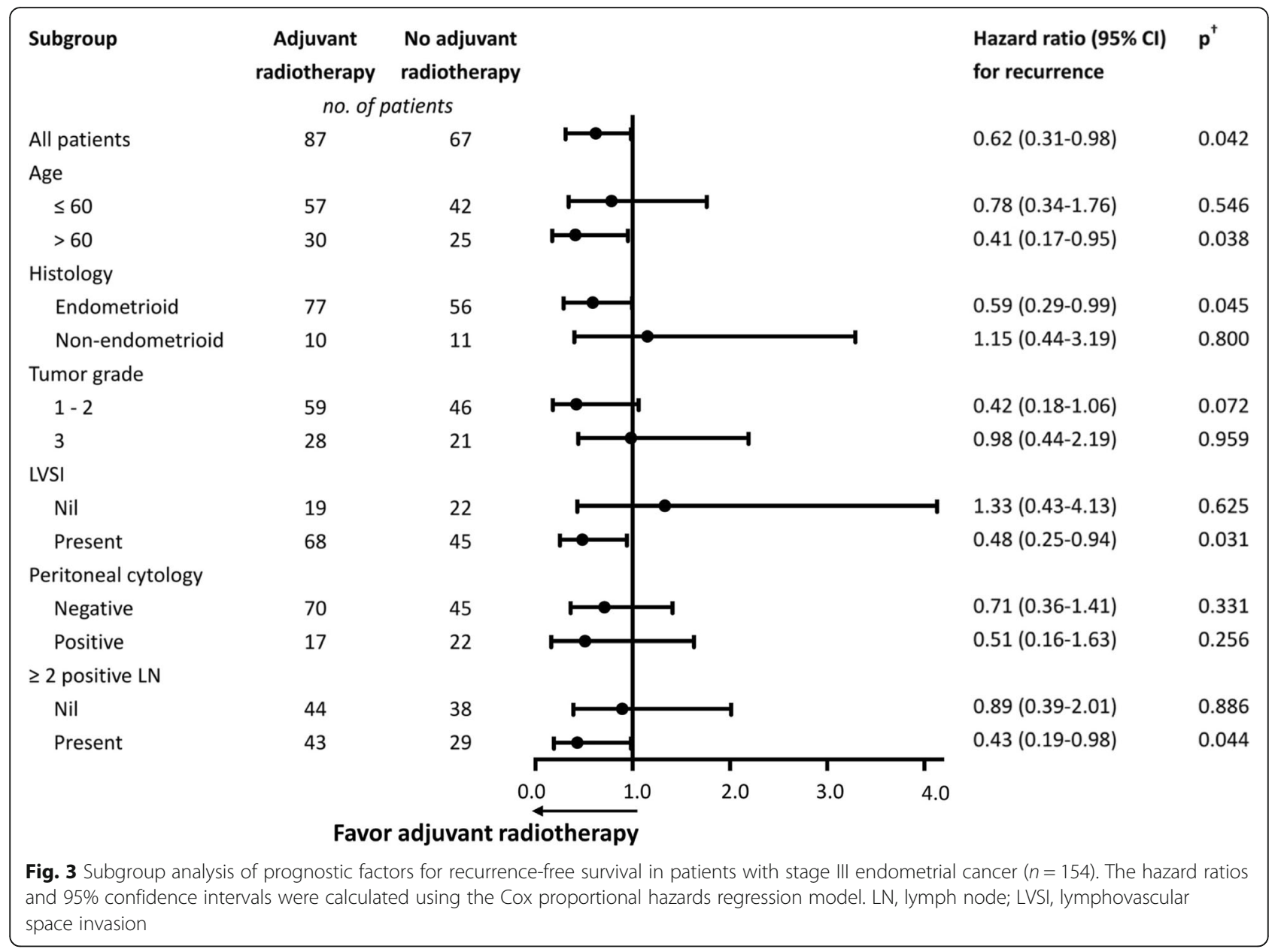

among patients aged $>60$ years $(p=0.038)$ as well as those with endometrioid histology $(p=0.045)$, lymphovascular space invasion $(p=0.031)$, and $\geq 2$ positive lymph nodes $(p=0.044)$. However, adjuvant radiotherapy did not significantly reduce the risk of recurrence among patients with grade 3 tumors or those with positive peritoneal washing cytology.

\section{Discussion}

To our knowledge, our study is the first to emphasize the value of modern adjuvant radiotherapy, including IMRT and VMAT, on clinical survival specifically in women with optimally resected stage III endometrial cancer. We found that modern adjuvant radiotherapy alone or combined with chemotherapy may be beneficial for patients aged $>60$ years as well as those with endometrioid histology, lymphovascular space invasion, and $\geq$ 2 positive lymph nodes.

In our present study, adjuvant radiotherapy by modern radiotherapy techniques (IMRT and VMAT) led to low incidence of acute (25\%) and chronic (7\%) grade $\geq 2$ gastrointestinal toxicity; by contrast, the aforementioned studies on conventional radiotherapy reported high acute $(45 \%)$ and late $(23 \%)$ grade $\geq 2$ gastrointestinal toxicity [11]. These data demonstrated that the use of modern radiotherapy techniques (IMRT and VMAT) effectively reduced the dose of radiation in the bowel with a clinical benefit of decreasing acute and late gastrointestinal toxicity $[17,20]$.

The use of adjuvant radiotherapy for patients with lymph node-positive endometrial cancer is under investigation. Increasing evidence shows that adjuvant radiotherapy improves locoregional control and (potentially) survival not only in patients with early-stage endometrial cancer who have high-risk features, but also in those with advanced-stage endometrial cancer [6, 24-26]. Our data are consistent with previously published studies that showed adjuvant radiotherapy to be effective for patients with node-positive endometrial cancer. Schmid et al. [26], who analyzed 943 patients with (surgically staged) stage III node-positive uterine cancer from the United States' Surveillance Epidemiology and End Results database, concluded that adjuvant radiotherapy was associated with a significant survival benefit in women with node-positive endometrioid uterine cancers. Brown et al. [24] investigated 116 patients with (surgically 
staged) stage IIIC endometrial cancer and demonstrated the benefits of adjuvant radiotherapy for those with positive para-aortic lymph nodes and those with $\geq 2$ positive nodes. Secord et al. [25] analyzed 265 patients with stage IIIC disease and concluded that adjuvant radiotherapy alone or combined with chemotherapy improved survival. IMRT and VMAT allow for simultaneous integral boosts to the extranodal extension areas or enlarged unresected lymphadenopathies, and provide accurate positioning and robust radiation doses that produce improved locoregional control and longer disease-free survival.

Several published data suggest that patients with stage III endometrial cancer might benefit from a combination radiotherapy-chemotherapy approach. According to Wang et al. [3], who analyzed 8738 patients from the United States' National Cancer Database with (surgically staged) stage III uterine cancer who had received adjuvant treatments, concluded that chemoradiotherapy was associated with superior survival outcomes compared to monotherapy. Studies by Kuku et al. [4], Marchetti et al. [5], and Lee et al. [27] of 90, 82, and 66 patients with stage IIIC endometrioid adenocarcinoma, respectively, all concluded that sequential chemoradiotherapy improved patient survival. Most of these data were retrospective and the modalities used were conventional two- or three-dimensional conformal radiotherapy that was associated with insufficient target coverage, inadequate sparing of the organs at risk, and inability to provide a simultaneous integrated boost to the extranodal extension areas or to enlarged unresected lymphadenopathies; all these limitations would compromise the efficacy of radiotherapy.

The prospective GOG 122 randomized controlled trial investigated surgically resected patients with FIGO stage III or IV who received adjuvant whole abdomen irradiation or adjuvant chemotherapy [28] and concluded that chemotherapy was superior to whole abdomen irradiation given that the 5 -year OS was $50 \%$ in the former group and 38\% in the latter group. The GOG 258 trial further investigated patients with surgically resected FIGO stage III or IV who received adjuvant chemotherapy either with or without radiotherapy [9], and concluded that chemotherapy plus radiotherapy (5-year OS, $59 \%$ ) did not produce superior outcomes than did chemotherapy alone (5-year OS, 58\%). However, the radiotherapy administered in the aforementioned trials was conventional and had suboptimal sparing of the organs at risk; this may have underestimated the role of adjuvant radiotherapy. The PORTEC-3 trial investigated radiotherapy alone or with chemotherapy in patients with surgically resected high-risk endometrial cancer [8] and concluded that stage III patients might benefit from the combined approach, as the 5-year OS was 78\% in the chemoradiotherapy group and was $68 \%$ in the radiotherapy-only group. Our data were in agreement with those of the PORTEC-3 trial, as we showed that modern adjuvant radiotherapy alone or combined with chemotherapy may benefit patients with stage III endometrial cancer.

A therapeutic alternative is brachytherapy, however, although it is considered an integral part of treatment for gynecologic cancers, modern radiotherapy methods (IMRT and VMAT) have shown advantages as substitutes, where brachytherapy is neither possible nor available. In patients who underwent hysterectomy but are contraindicated for, or decline brachytherapy on the vaginal cuff, a simultaneous integrated boost with IMRT or VMAT can be proposed as a therapeutic alternative $[29,30]$. Furthermore, given the advantage of the noninvasiveness of radiotherapy generally, modern radiotherapy is considered a winning treatment option for elderly patients or patients unfit for surgery or chemotherapy in order to improve their survival and quality of life [16, 31]. In addition, the advantage of precise high dose targeting with minimum harm to the neighboring organs by IMRT and VMAT allows for a risk-adapted dose prescription, where a boost dose to documented extranodal extension or enlarged unresected lymphadenopathy is technically feasible and clinically effective for higher locoregional control [17]. This information echoes with our results that older patients as well as those with endometrioid histology, lymphovascular space invasion, and $\geq 2$ positive lymph nodes benefited the most from adjuvant radiotherapy.

Although accumulating studies of modern radiotherapy methods (IMRT and VMAT) show their superiority to conventional radiotherapy techniques, their limitations include high cost, increased staff burden, longer time for treatment planning, and risks of marginal misses [12]. Our study had several limitations, including its retrospective design. The patient population was heterogeneous in terms of stage subgrouping, tumor histology, and characteristics. Patients did not receive uniform chemotherapy nor multimodality treatments. The median follow-up duration was 44 months; as such, longer follow-up times may be required to further investigate patient outcomes.

\section{Conclusion}

Our data support the indication of adjuvant treatment for women with optimally resected stage III endometrial cancer despite the abovementioned limitations. In the era of modern radiotherapy, adjuvant radiotherapy alone or in combination with chemotherapy was found to be associated with improved survival and may be particularly beneficial for patients $>60$ years of age as well as those with endometrioid histology, lymphovascular space invasion, and $\geq 2$ positive lymph nodes. These findings may be further investigated in prospective clinical trials. 


\section{Supplementary information}

Supplementary information accompanies this paper at https://doi.org/10. 1186/s13014-020-01523-5.

Additional file 1: Supplementary Figure 1. Survival in patients with optimally resected stage III endometrial cancer based on whether or not adjuvant treatment was performed. Recurrence-free survival (RFS) (a) and overall survival (OS) (b) of patients based on whether or not adjuvant treatment was performed. $p$-values were determined using Kaplan-Meier log-rank tests.

\section{Abbreviations}

EBRT: External beam radiotherapy; IMRT: Intensity-modulated radiation therapy; VMAT: Volumetric modulated arc therapy; FIGO: International Federation of Gynecology and Obstetrics; RFS: Recurrence-free survival; OS: Overall survival

\section{Acknowledgments}

We thank the staff of the Core Labs, Department of Medical Research National Taiwan University Hospital, for their technical support.

\section{Authors' contributions}

$J$ L-Y Chen and Y-S Huang were responsible for study design and plan, literature collection, data management and interpretation, statistical analysis, and manuscript writing. J L-Y Chen, Y-S Huang, and C-Y Hsu were responsible for statistical analysis and interpretation of the statistical findings. J L-Y Chen, C-Y Hsu, K-H Lan, W-F Cheng, and S-H Kuo contributed to the patient cohort and clinical data. J L-Y Chen, Y-S Huang, C-Y Hsu, and K-H Lan were responsible for the data management. J L-Y Chen, W-F Cheng, and S-H Kuo planned, designed, and coordinated the study over the entire period and wrote the manuscript. All authors read and approved the final manuscript.

\section{Funding}

This work was supported by the National Taiwan University Hospital (NTUH) and the Ministry of Science and Technology (MST, Taiwan).

\section{Availability of data and materials}

The data that support the findings of this study were used under license from the National Taiwan University Hospital Healthcare System database, and so are not publicly available. Limited data are available from the corresponding author on reasonable request.

\section{Ethics approval and consent to participate}

All procedures were in accordance with the ethical standards of the institutional and/or national research committee and with the 1964 Helsinki Declaration and its later amendments or comparable ethical standards. The study was approved by National Taiwan University Hospital Research Ethics Committee (approval number: 201910019RINA)

\section{Consent for publication}

Not applicable.

\section{Competing interests}

The authors declare that they have no competing interests.

\section{Author details}

'Department of Radiology, National Taiwan University College of Medicine, No. 7, Chung-Shan S. Rd, Taipei, Taiwan. ²Division of Radiation Oncology, Department of Oncology, National Taiwan University Hospital, Taipei, Taiwan. ${ }^{3}$ Department of Medical Imaging, National Taiwan University Hospital Hsin-Chu Branch, Hsinchu, Taiwan. ${ }^{4}$ National Taiwan University Cancer Center, National Taiwan University College of Medicine, Taipei, Taiwan. ${ }^{5}$ Department of Medical Imaging, National Taiwan University Hospital, Taipei, Taiwan. ${ }^{6}$ Department of Medical Imaging, National Taiwan University Hospital Yun-Lin Branch, Yunlin, Taiwan. ${ }^{7}$ Department of Obstetrics and Gynecology, National Taiwan University Hospital, Taipei, Taiwan.
Received: 28 January 2020 Accepted: 24 March 2020

Published online: 06 April 2020

\section{References}

1. Lortet-Tieulent J, Ferlay J, Bray F, Jemal A. International patterns and trends in endometrial cancer incidence, 1978-2013. J Natl Cancer Inst. 2018;110(4): 354-61.

2. Tai YJ, Hsu HC, Chiang YC, Chen YL, Chen CA, Cheng WF. Impact of adjuvant modalities on survival in patients with advanced stage endometrial carcinoma: a retrospective analysis from a tertiary medical center. Int J Environ Res Public Health. 2019;16(14):E2561-72.

3. Wang CJ, Christie A, Folkert MR, Xie XJ, Albuquerque K. Value of combined adjuvant chemotherapy and radiation on survival for stage III uterine cancer: is less radiation equal to more? J Gynecol Oncol. 2018;29(4):e49.

4. Kuku S, Williams M, McCormack M. Adjuvant therapy in stage III endometrial cancer: treatment outcomes and survival. A single-institution retrospective study. Int J Gynecol Cancer. 2013;23(6):1056-64.

5. Marchetti C, Pisano C, Mangili G, Lorusso D, Panici PB, Silvestro G, et al. Use of adjuvant therapy in patients with FIGO stage III endometrial carcinoma: a multicenter retrospective study. Oncology. 2011:81(2):104-12.

6. Milgrom SA, Kollmeier MA, Abu-Rustum NR, Tew WP, Sonoda Y, Barakat RR, et al. Postoperative external beam radiation therapy and concurrent cisplatin followed by carboplatin/paclitaxel for stage III (FIGO 2009) endometrial cancer. Gynecol Oncol. 2013;130(3):436-40.

7. Deleon MC, Ammakkanavar NR, Matei D. Adjuvant therapy for endometrial cancer. J Gynecol Oncol. 2014:25(2):136-47.

8. de Boer SM, Powell ME, Mileshkin L, Katsaros D, Bessette P, Haie-Meder C, et al. Adjuvant chemoradiotherapy versus radiotherapy alone in women with high-risk endometrial cancer (PORTEC-3): patterns of recurrence and post-hoc survival analysis of a randomised phase 3 trial. Lancet Oncol. 2019; 20(9):1273-85.

9. Matei D, Filiaci V, Randall ME, Mutch D, Steinhoff MM, DiSilvestro PA, et al. Adjuvant chemotherapy plus radiation for locally advanced endometrial cancer. N Engl J Med. 2019;380(24):2317-26

10. Chen JL, Huang YS, Kuo SH, Hong RL, Ko JY, Lou PJ, et al. Intensitymodulated radiation therapy achieves better local control compared to three-dimensional conformal radiation therapy for T4-stage nasopharyngeal carcinoma. Oncotarget. 2017;8(8):14068-77.

11. Klopp AH, Yeung AR, Deshmukh S, Gil KM, Wenzel L, Westin SN, et al. Patient-reported toxicity during pelvic intensity-modulated radiation therapy: NRG oncology-RTOG 1203. J Clin Oncol. 2018;36(24):2538-44.

12. Citrin DE. Recent developments in radiotherapy. N Engl J Med. 2017;377(11): 1065-75.

13. Peters $\mathrm{S}$, Schiefer $\mathrm{H}$, Plasswilm L. A treatment planning study comparing Elekta VMAT and fixed field IMRT using the varian treatment planning system eclipse. Radiat Oncol. 2014:9:153.

14. Beadle BM, Liao KP, Elting LS, Buchholz TA, Ang KK, Garden AS, et al. Improved survival using intensity-modulated radiation therapy in head and neck cancers: a SEER-Medicare analysis. Cancer. 2014;120(5):702-10.

15. Chun SG, Hu C, Choy H, Komaki RU, Timmerman RD, Schild SE, et al. Impact of intensity-modulated radiation therapy technique for locally advanced non-small-cell lung cancer: a secondary analysis of the NRG oncology RTOG 0617 randomized clinical trial. J Clin Oncol. 2017;35(1):56-62.

16. Fiorentino A, Gregucci F, Mazzola R, Figlia V, Ricchetti F, Sicignano G, et al. Intensity-modulated radiotherapy and hypofractionated volumetric modulated arc therapy for elderly patients with breast cancer: comparison of acute and late toxicities. Radiol Med. 2019;124(4):309-14

17. Mazzola R, Fersino S, Aiello D, Gregucci F, Tebano U, Corradini S, et al Linac-based stereotactic body radiation therapy for unresectable locally advanced pancreatic cancer: risk-adapted dose prescription and imageguided delivery. Strahlenther Onkol. 2018;194(9):835-42.

18. Pecorelli S. Revised FIGO staging for carcinoma of the vulva, cervix, and endometrium. Int J Gynaecol Obstet. 2009;105(2):103-4.

19. National Comprehensive Cancer Network. Uterine Neoplasms (Version 5. 2019) 2019 [cited 2019 December 23]. Available from: https://www.nccn org/professionals/physician_gls/pdf/uterine.pdf. Accessed 1 March 2020

20. Chen JL, Wang MC, Huang YS, Huang CY, Pan CK, Hsu CY, et al. Extendedfield bone marrow sparing radiotherapy for primary chemoradiotherapy in cervical cancer patients with Para-aortic lymphadenopathy: volumetricmodulated arc therapy versus helical Tomotherapy. J Xray Sci Technol. 2020; 28(1):111-24 
21. Chan P, Yeo I, Perkins G, Fyles A, Milosevic M. Dosimetric comparison of intensity-modulated, conformal, and four-field pelvic radiotherapy boost plans for gynecologic cancer: a retrospective planning study. Radiat Oncol. 2006;1:13.

22. Chen JL, Cheng JC, Kuo SH, Chen CA, Lin MC, Huang CY. Outcome analysis of cervical adenosquamous carcinoma compared with adenocarcinoma. Acta Obstet Gynecol Scand. 2012;91(10):1158-66.

23. Chen JL, Huang CY, Huang YS, Chen RJ, Wang CW, Chen YH, et al. Differential clinical characteristics, treatment response and prognosis of locally advanced adenocarcinoma/adenosquamous carcinoma and squamous cell carcinoma of cervix treated with definitive radiotherapy. Acta Obstet Gynecol Scand. 2014;93(7):661-8.

24. Brown AP, Gaffney DK, Dodson MK, Soisson AP, Belnap TW, Alleman K, et al. Survival analysis of endometrial cancer patients with positive lymph nodes. Int J Gynecol Cancer. 2013:23(5):861-8.

25. Secord AA, Geller MA, Broadwater G, Holloway R, Shuler K, Dao NY, et al. A multicenter evaluation of adjuvant therapy in women with optimally resected stage IIIC endometrial cancer. Gynecol Oncol. 2013;128(1):65-70.

26. Schmid S, Hsu IC, Hu JM, Sherman AE, Osann K, Kapp DS, et al. Adjuvant radiation therapy in stage III node-positive uterine cancer. Gynecol Oncol. 2009;115(2):239-43.

27. Lee $\amalg$, Viswanathan AN. Combined chemotherapy and radiation improves survival for node-positive endometrial cancer. Gynecol Oncol. 2012;127(1): 32-7.

28. Homesley HD, Filiaci V, Gibbons SK, Long HJ, Cella D, Spirtos NM, et al. A randomized phase III trial in advanced endometrial carcinoma of surgery and volume directed radiation followed by cisplatin and doxorubicin with or without paclitaxel: a gynecologic oncology group study. Gynecol Oncol. 2009;112(3):543-52.

29. Alongi F, Mazzola R, Ricchetti F, Fersino S, Levra NG, Fiorentino A, et al. Volumetric-modulated arc therapy with vaginal cuff simultaneous integrated boost as an alternative to brachytherapy in adjuvant irradiation for endometrial cancer: a prospective study. Anticancer Res. 2015;35(4): 2149-55.

30. Mahmoud O, Kilic S, Khan AJ, Beriwal S, Small W Jr. External beam techniques to boost cervical cancer when brachytherapy is not an optiontheories and applications. Ann Transl Med. 2017;5(10):207.

31. Gregucci F, Fiorentino A, Corradini S, Figlia V, Mazzola R, Ricchetti F, et al. Linac-based radiosurgery or fractionated stereotactic radiotherapy with flattening filter-free volumetric modulated arc therapy in elderly patients : a mono-institutional experience on 110 brain metastases. Strahlenther Onkol. 2019;195(3):218-25.

\section{Publisher's Note}

Springer Nature remains neutral with regard to jurisdictional claims in published maps and institutional affiliations.

Ready to submit your research? Choose BMC and benefit from:

- fast, convenient online submission

- thorough peer review by experienced researchers in your field

- rapid publication on acceptance

- support for research data, including large and complex data types

- gold Open Access which fosters wider collaboration and increased citations

- maximum visibility for your research: over $100 \mathrm{M}$ website views per year

At BMC, research is always in progress.

Learn more biomedcentral.com/submissions 